\title{
Current Advances and Prospects on Implementation of Highly Sensitive Aptamer-based Dual System for Melamine Detection: New Promising Tool of Great Affinity
}

\author{
Mukama Omar ${ }^{1,2}$, Ndikubwimana Jean de Dieu', Muhammad Shamoon ${ }^{3}$ and Byong H Lee ${ }^{4^{\star}}$ \\ ${ }^{1}$ Key Laboratory of Carbohydrate Chemistry and Biotechnology, School of Biotechnology, Jiangnan University, Wuxi 214122, P.R. China \\ ${ }^{2}$ Department of Applied Biology, College of Science and Technology, Avenue de l'armée, P.O. Box: 3900 Kigali, Rwanda \\ ${ }^{3}$ The synergistic Innovation Center of Food Safety and Nutrition, State Key Laboratory of Food Science and Technology, Jiangnan University, Wuxi 214122, P.R. China \\ ${ }^{4}$ Department of Food Science/Agricultural Chemistry, McGill University, Saint-Anne-de-Bellevue, Qc H9X3V9, Canada
}

\begin{abstract}
Much attention has been devoted to melamine (MA) analysis in food products in accordance with the food safety standards. Aptamer-based analytical techniques thrived with the improvements in latest tools, analytical reagents and methods and most importantly iterative in vitro selection process known as systematic evolution of ligands by exponential enrichment (SELEX). Aptamer-based techniques possess very high affinity and specificity towards contaminants and play a pivotal role in MA detection. However, success depends on the starting aptamer, selection of appropriate nanoparticle, target molecule(s) and characterization of advances in aptamer selection, strategies of preparing, treating the nanoparticles analytical system methods. Current review has focused to elaborate the key recent innovation in aptamer-based dual system construction for MA detection. We have also highlighted the promising types of aptamer-conjugated nanomaterial for the specific recognition of some other potential adulterants and food hazards. Finally, we proposed future directives in developing novel aptamer and further condition optimizations that could give high-throughput food-safety analysis method and melamine "zero tolerance" towards food safety incidents.
\end{abstract}

Keywords: Aptamer; SELEX; Nanoparticles; Melamine; Detection

Abbreviations: CDC: Centers For Disease Control Prevention; Agnps: Silver Nanoparticles; TSE: Transmissible Spongiform Encephalopathy; Aunps: Gold Nanoparticles; HPLC: HighPerformance Liquid Chromatography; CE: Capillary Electrophoresis; AFM: Atomic Force Microscopy; CTA: Chromotropic Acid; PDDA: Polydiallyldimethylammonium Chloride; GC: Gas Chromatography; ELISA: Enzyme-Linked Immunosorbent Assay; Haucl4: Chloroauric Acid; SELEX: Systematic Evolution of Ligands By Exponential Enrichment; LOD: Limit of Detection; STM: Scanning Tunneling Microscope; MA: Melamine; SPM: Scanning Probe Microscopy; MS: Mass Spectrometry; SPR: Surface Plasmon Resonance Assay; Prp: Prion Protein; SERS: Surface Enhanced Raman Spectroscopy; SAA: Salfanilic Acid; ELAAS: Enzyme-Linked Aptamer Assays; UTR: Untranslated Region

\section{Introduction}

Recently, melamine (MA) has become one of the major global food safety concerns being notorious for its effects on human and animal health. Owing to its water-repellent, shrink-resistant, stain-repellent and fire-retardant properties, the role of MA has gained a significant consideration in manufacturing of tableware, industrial coatings, paints, adhesives, glues paper, floor tiles, paperboard and plastic packages. It has common structure with triazine family where some of the members serve as herbicides. MA is a non-registered fertilizer in the U.S. but it is still practiced as a fertilizer in some other parts of the world [1]. In 2007, Food and Drug Administration (FDA) strictly prohibited MA as a source of non-protein nitrogen in food or feed even if it cannot solely cause side effects on animals like cats and dogs. However, when combined with its derivative cyanuric acid through gut microbiota mediation (Figure 1) (e.g. Klebsiella terrigena), it forms insoluble crystals in pets leading to renal failure [2]. The hallmark of kidney stones is renal colic, hematuria, little or no urine, kidney infection, and/or high blood pressure. The cases of pediatric ailments like kidney stones caused by MA has been reported in nearly 300,000 infants and deaths of six by the Pediatric Environmental Health Specialty Units (PEHSU) [3]. In 2008, the Centers for Disease Control Prevention (CDC) has also found MA in Chinese-manufactured infant formula [4]. To date, MA detection has extended beyond the milk products to meat, poultry, eggs and various vegetables. MA deposit in eggs from poultry fed with MA contaminated feed [5]. MA nitrogen content is above $65 \%$ by weight, thus it is use as food adulterant and traditional nitrogen-based tests of Kjeldahl and Dumas methods lack accuracy in discriminating between the proteinous and MA nitrogen [6]. Safety limit of MA set by UN food standard commission in food and beverages is 2.5 and $1 \mathrm{ppm}$ respectively with exception of infant formula $[7,8]$; and this has led to search for the fast and accurate detection methods. Several analytical techniques are currently available such as fluorescence-based techniques [9], scanning probe microscopy (SPM, AFM, STM), surface enhanced raman spectroscopy (SERS) [10], electrochemical [11], surface plasmon resonance assay (SPR) [12] and impedance spectroscopy for MA [13]. Development of analytical techniques such as liquid chromatography [14], liquid chromatography-tandem mass spectrometry (LC-MS/MS) have been used for MA screening and confirmation due to their good selectivity and detection limits [15-17]. HPLC with fluorescence detection of MA [18], LC-Raman spectroscopy [19], capillary zone electrophoresis (CE) have been also utilized [20]. Despite accuracy of instrumental techniques such as HPLC, GC, and CE; they are time-consuming and

*Corresponding author: Byong $\mathrm{H}$ Lee, Distinguished Professor, Department of Food Science/Agricultural Chemistry, McGill University, Saint-Anne-de-Bellevue, Qc H9X3V9, Canada, Tel: 82-33-250-6453; E-mail: byonghlee@gmail.com

Received March 17, 2015; Accepted April 27, 2015; Published April 30, 2015

Citation: Omar M, Dieu NJD, Shamoon M, Lee BH (2015) Current Advances and Prospects on Implementation of Highly Sensitive Aptamer-based Dual System for Melamine Detection: New Promising Tool of Great Affinity. J Bioprocess Biotech 5 : 220 doi:10.4172/2155-9821.1000220

Copyright: ( 2015 Omar M, et al. This is an open-access article distributed under the terms of the Creative Commons Attribution License, which permits unrestricted use, distribution, and reproduction in any medium, provided the original author and source are credited. 
expensive [21]. Furthermore, in detection of melamine in pet food samples, an inhibition ELISA was developed based on the monoclonal antibody that give efficient standard linear curve ranging from 0.03 to $9 \mathrm{ng} / \mathrm{l}$ with a limit of detection (LOD) $0.01 \mathrm{ng} / \mathrm{ml}$ and assay sensitivity $0.35 \mathrm{ng} / \mathrm{ml}$ [22]. However, the above mentioned techniques require more time of development and cannot be compared to aptamersbased techniques. The latter possess the ability to target molecules for which instrumental techniques and antibodies are not well suited with equal high specificity and affinity. Currently, aptamer-coupled techniques such as immunological (nano-ELAAS) [23], fluorescent chemicals (perlene) [24], nanoparticles (NPs) [25,26], electrochemical $[27,28]$ and colorimetric aptamers [29] are available in form of biosensors. The use of nucleic acids bio-detectors 'Aptamers' showed high ability to recognize specific targets with great affinity, stability, quick, cheap and easy to develop using SELEX [30] or automated in vitro selection that reduces the duration of a selection process from several weeks to three days [31]. Aptamer is derived from the Latin word "aptus" meaning "to fit" because of its strong binding to specific targets based on structural conformation. More interestingly, its combination to colorimetric nanomaterials, gives naked-eye visual detection. In this review, we have elucidated recent implementation of nanomaterial-nucleic acid (aptamers) dual system construction in bioassays to detect the infinitesimal amount of MA in foodstuffs added either non-intentionally (e.g. melamine-formaldehyde resin migration from tableware to food or beverages) or intentionally as an adulterant.

\section{Medical Significance of Melamine}

Analytical techniques for MA detection are highly needed in biomedical and clinical field due to frequent use of plastic tableware, water dispensers and other similar sources of MA. The HPLC analysis showed that $20.78 \%$ of breast milk samples contained MA levels between 10.09 and $76.43 \mathrm{ng} / \mathrm{l}$ [32]. Despite the LOD was below than that established by WHO, fast diagnosis for MA is still a significant concern to prevent the acute renal failure development (Figure 1). Continuous consumption of MA in low dose may also lead to urolithiasis and nephrolithiasis [33], hippocampal synaptic plasticity and spacial memory [34]. Currently, Yin et al. revealed the effects of MA on mice spleen lymphocytes by decreasing CD4+/CD $8+$ in presence or absence of MA-cyanuric acid complex formation, suggesting that MA may act as inducer and suppressor of expression of Bax and Bcl-2, respectively [35]. As a consequence of this, Aptamer and nanoparticles assay in detection of molecules such as MA, DNA and proteins may prove a new tool of detection.

\section{Selection of Aptamer Probes}

RNA has ability not only in coding for proteins but also in catalyzing reactions, binding other RNAs, proteins or specifically can bind to a target ligand(s) [30]. Aptamers are obtained from a large random sequence pool containing about 1013-1015 single-strand RNAs composed of a random sequence region flanked by a binding site. Incubation of these oligonucleotides with the single target or a large variety of targets molecule(s) results binding with high affinity and specificity in three-dimensional shape [36]. Few nucleic acids bind to the target are considered as aptamers. This step is followed by washing which help to filter out unbound nucleic acids. Aptamers are separated from the target by elution and PCR amplification of bound nucleic acids is done to create a new library. The selected sequences used in a new round of SELEX for further optimization (Figure 2). The variant SELEX exists to enhance oligonucleotides conformation stability or resistance to nucleases depending on target molecule. Some of them are genomic SELEX or cDNA-SELEX [37], photo SELEX
[38], covalent-SELEX or cross-linking SELEX [39-41], multistage SELEX [42] used for starting pool rationalization or improve aptamer selectivity using negative SELEX protocol or counter SELEX, and deconvolution SELEX or subtractive SELEX. These help to remove undesirable adsorbed oligonucleotides from the poolby matrixes that used for immobilizing the targets and distinction of similar structures [43]. In addition, in order to enhance targets applicability, other variants SELEX used are: Whole bacteria-SELEX [43], blended SELEX [44], TECS-SELEX [45], complex target SELEX [46], toggle SELEX targetswitching [47], expressions cassette also known as SELEX-SAGE [48], the mirror-image SELEX or Spiegelmer technology [49], whole cellSELEX [50]. Efficacy is the prerequisite for any aptamer-based design; several SELEX strategies such as non-SELEX or NECEEM-SELEX, CE-SELEX [51], microfluidic SELEX [52], HTS-SELEX or automated SELEX [53], FluMag-SELEX [54], in silico SELEX [55] are applicable. However, currently improvements in SELEX have been made. For example: SELEX process needs a PCR step, the randomized region of the oligonucleotide libraries must be flanked by two fixed primers binding sequences. It has been designed an in vitro selection novel type of dual RNA library for mirror-image peptides detection like Ghrelin. It carries fixed sequences which constrains the oligonucleotides into a partially double-stranded structure that allows primer less selection in order to minimize the primer binding sequences to be part of the target-binding motif [56]. Moreover, DNA aptamers with only natural bases can often lack the desired specificity and binding affinity to target proteins compared to aptamers containing recombinant bases. Thus, over 100-fold affinity DNA aptamers have been developed using expanded genetic alphabet of nucleic acid (use of unnatural libraries bases). The DNA aptamer possessing the four natural nucleotides and unnatural nucleotides against two human target proteins, interferon- $\gamma$ (IFN- $\gamma$ ) and vascular endothelial cell growth factor-165 (VEGF-165) that bind with the dissociation constant of $0.038 \mathrm{nM}$ and $0.65 \mathrm{pM}$, respectively has also been developed in the same fashion [57].

For MA detection, the most commonly used aptamers are in form of poly-Ts aptamer (ssDNA) with purified sequence 5'TTTT TTTTTTTTTTTTTTTTTTTTTTTTTTT [58]. Yun et al. used the selected MA aptamer 5'-TTTTTTTTTTTTTTTTTTTT-3' to detect melamine in milk sample. This aptamer has potential to protect gold nanoparticles under high salt concentration conditions by averting the immediate colour change of AuNPs upon addition of MA because the latter combine competitively with aptamer [59]. Thus, the well selected aptamer shows efficiency in stabilizing nanomaterials and consequently gives accurate results (Figure 2).

\section{Gold Nanoparticles for MA Detection}

The gold, silver and quantum dots nanoparticles have emerged as new tools that ensure the food safety via providing fast and reliable detection approach for targets with simplicity, lower cost compared with immunoassay-based and chromatography based methods. Gold nanoparticles (AuNPs) are synthesized using sodium citrate reduction method [60]. This method yields particles with desired dimensions by changing the gold precursor salt and sodium citrate molar ratio. It works both as a reducing agent for nucleation of AuNPs and as stabilizing agent by coating the nanoparticles' surface and inhibiting their aggregation in solution. When MA is introduced in the system, hydrogen bonds formation between MA amine groups and citrate ions decrease the electrostatic repulsion between individual AuNPs and results in the AuNPs aggregation. The major advantages are based on the facts that no sophisticated apparatus is needed, rapid and nakedeye color change observation, giving a precise quantification of MA 
when analyzed on UV-Vis spectrometer. For example, well-dispersed AuNPs solution is red, whereas aggregated AuNPs appear in blue or purple colour [61]. However, other methods of AuNPs synthesis are used because sodium citrate method provides small size nanoparticles concentration. AuNPs are synthesized from concentrated $\mathrm{HAuCl}_{4}$ via addition of sodium hydroxide, $\mathrm{pH}$ and temperature control [62]. MA was detected using AuNPs with a LOD $0.4 \mathrm{mg} / \mathrm{L}$ displayed in 12 min including sample preparation [63]. The developed gold nanoparticle-based kit for MA detection in milk products reached working range of 1-120 mg/l improving time of detection about 10 min including sample pretreatment [64]. However, Cao et al. found that hydrogen-bonding can induce colorimetric detection of MA by label free, and nonaggregation-based AuNPs probe prepared using 3,5-dihydroxybenzoic acid (DBA) reducer interact with MA through strong hydrogen-bonding interaction. Consequently, the melamine can hinder nanoparticles generation resulting in color change from purple to yellow green with gradual increase of melamine concentration. The plasmon absorbance of the formed AuNPs allows the quantitative detection of MA with MA concentration sensitivity ranging from $1 \times$ $10^{-9} \mathrm{M}$ to $1 \times 10^{-5} \mathrm{M}$, a linear coefficient of 0.993 and high selectivity to melamine with a low detection limit of $8 \times 10^{-10} \mathrm{M}$ [65]. As Media pH and reaction time are major influencing factors of AuNPs aggregation, Naveen et.al recently improved citrate reduction method [63] by preparing AuNPs with $5 \mathrm{ml}$ of $38.8 \mathrm{Mm}$ trisodium citrate, $8.0 \mathrm{NaOH}$ and further optimization in sample extraction and detection of MA. The AuNPs changes from its wine red color to blue or purple with MA detection down to a concentration of $0.05 \mathrm{mg} / \mathrm{l}$ determined by monitoring with the naked eyes or a UV-Vis spectrophotometer [66].

Although silver nanoparticles AgNPs are often used for MA detection, gold nanoparticles are more stable with less stringent requirements for storage and handling. In addition, during melamine detection, it has also been seen that AuNPs treated with citrate buffer induce highly an enhanced melamine signals in the Raman spectrum due to the formation of SERS "hot spots" caused by the ability of melamine to form AuNPs aggregates. The concentration range of 0.31-5.0 mg/l in milk with a limit of detection of $0.17 \mathrm{mg} / \mathrm{l}$ has been obtained in lower than 30 minutes [67]. Even though it improved time of detection, its LOD is of concern.

\section{Silver Nanoparticles for MA Detection}

Silver nanoparticles (AgNPs) can be prepared using borohydride reduction method [68], but some modifications improved the efficiency. Its detection principle consists of the ability of AgNPs to be stabilized and coated in aqueous solution with negatively-charged citrate ions [69] able to interact via Van der Waals' force and with the positively-charged MA to its three exocyclic amine group and a threenitrogen hybrid ring. This means, when MA is added to a solution of AgNPs, attachment occurs with result of AgNPs aggregation followed by color change. It is thought that either three exocyclic amine group or a three-nitrogen hybrid ring results the label free AgNPs aggregation [70]. Compared with gold nanoparticles, AgNPs have some advantages, such as lower cost of preparation, higher extinction coefficients relative to AuNPs of the same size. Therefore, they are used as calorimetric probes of MA detection and other hazards to assure food safety and protect public health. Despite some advantages of Ag NPs over AuNPs, several papers proposed AuNPs as preferred substrates for melamine detection since the color of the particles changes after the MAAuNPs interaction in solution. The use of label-free AgNPs detection of MA concentration in raw milk pretreated with chloroform and trichloroacetic acid to remove the protein and fat resulted a naked eye or a UV-Vis spectrophotometer yellow to red visible color change with detection limit of $2.32 \mu \mathrm{M} \sim 0.29 \mathrm{mg} / \mathrm{l}$, which is below the safety limit of $2.5 \mathrm{ppm}$ recommended in USA and EU. Whereas for infant formula in China, only $1 \mathrm{ppm}$ of MA is tolerated, thus it has an advantage over some methods used for MA detection but not conducive as AuNPs [71]. However, in one way, this method can be interfered by some amino acids and other positively charged molecules that can give false positive results due to some instability of prepared AgNPs. In another way, no MA-cyanuric acid aggregation, that can thus show no color display. Therefore, chromotropic acid (CTA) capping of AgNPs makes them to remain in stable and dispersed form. Once MA is added in nanomaterials solution, it will bind with CTA found on the CTAAgNPs complex through hydrogen bonding with high selectivity to its structural analogs like cyanulic acid. MA only can act as hydrogen donor to sulfur- groups of the CTA-capped AgNPs and thus induces aggregation accompanied by yellow to orange color change observed by naked eyes [72]. However, it is still require a method for detection of MA in food and beverages with high sensitivity. Thus, the same group of Fangying developed an AgNPs conjugated with sulfanilic acid (SAA), this combination of techniques significantly increased the sensitivity and selectivity of melamine in pretreated milk and only can melamine react with SAA-AgNPs followed by rapid aggregation due to the MA affinity towards the SAA functional groups. The selectivity of this method is below $50 \mathrm{nM}$ and LOD of $10.6 \mathrm{nM}$ which is not only low compared to the multiple existing methods used to detect MA, but also cheap with one sample treatment with analysis costs of $0.5 \$$ [73]. However, further meticulous research with an easy, cost-effective method lies ahead to achieve ultrasensitive and selective methods for screening MA level in food products. In the next section, we have focused on the aptamer-nanomaterial techniques and different treatment strategies as a potential solution to nanoparticles drawbacks mentioned above.

\section{Aptamer-Conjugated Nanoparticle Based Novel System for MA Detection}

Aptamer-modified AuNPs show high stability and selectivity compared to unmodified AuNPs in MA and other small molecules sensing [74]. Either ssDNA or RNA aptamers are used to label nanoparticles in the synthesis of dual system aptamer-NP probe for MA detection. The probe is stabilized at $\mathrm{pH} 6.6$ or $7.0 \mathrm{NaH}_{2} \mathrm{PO}_{4}$

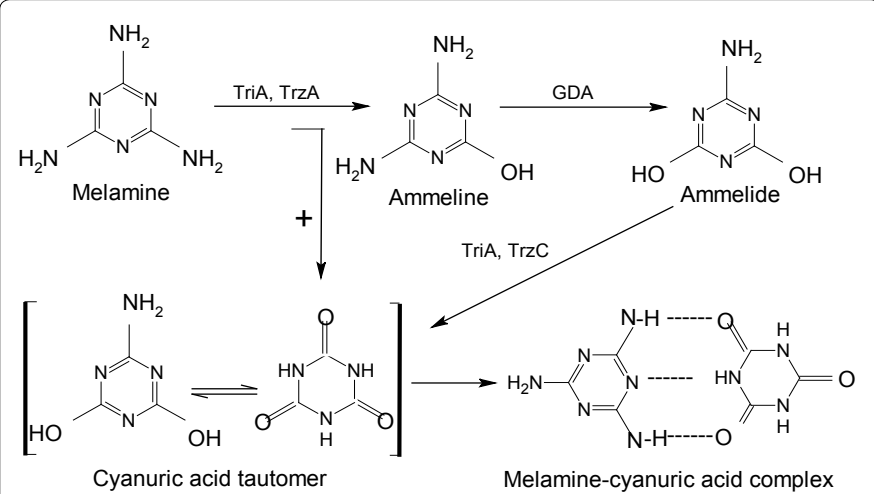

Figure 1: Melamine combined with its derivative product cyanuric acid to form melamine-cyanuric acid insoluble crystal complex that can cause renal failure (kidney stones, hematuria, high blood pressure etc.). This figure also depicts the gut microbiota mediation of enzymatic conversion of melamine to cyanuric acid. TriA stands for melamine deaminase, TrzA for S-trizine hydrolase, GD for Guanine deaminase, AtzC for $n$-isopropylammelide aminohydrolase and TrzC for Ammelide hydrolase. 


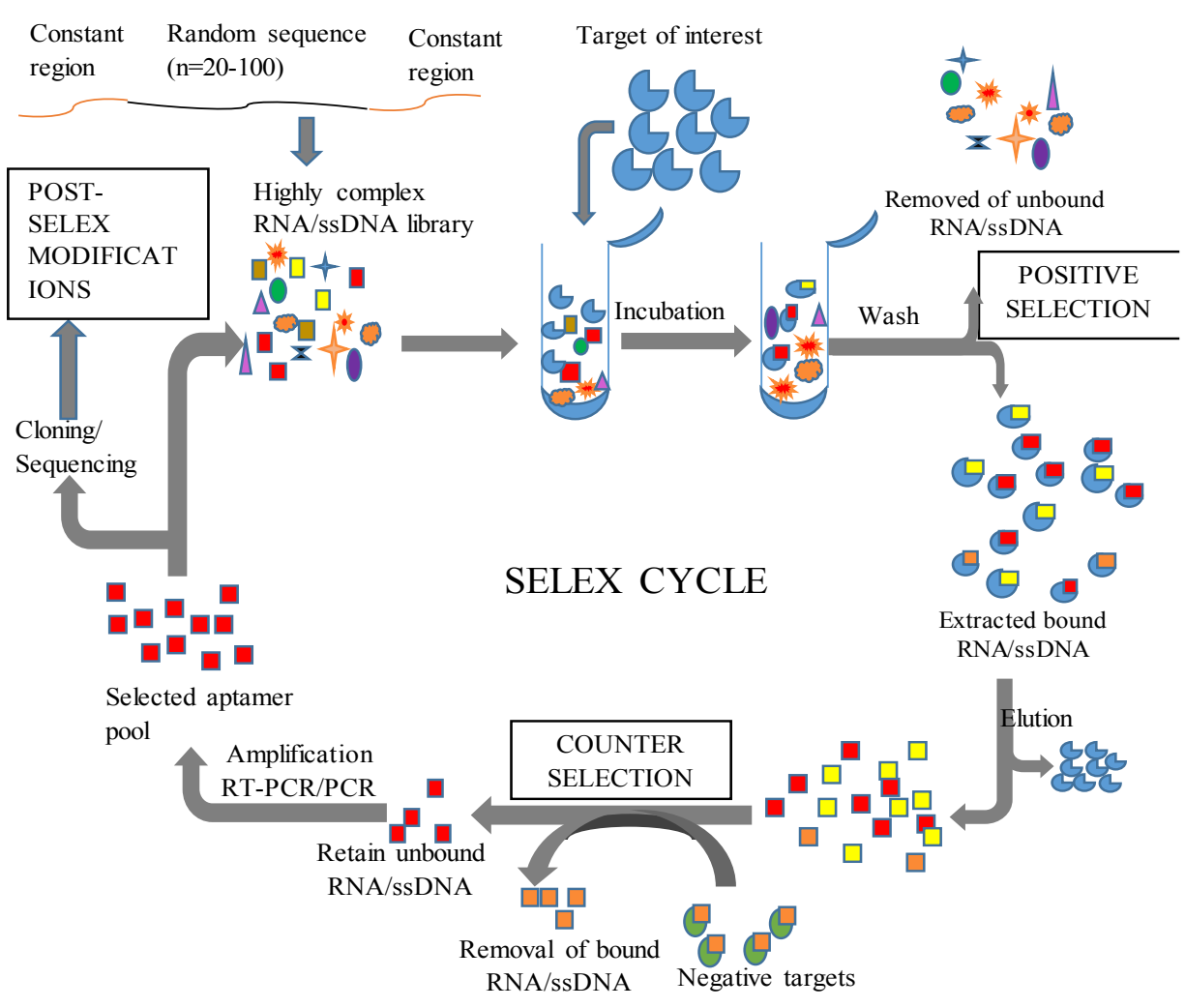

Figure 2: General principle of SELEX

$\mathrm{Na}_{2} \mathrm{HPO}_{4}$ buffer solutions and in high concentrated electrolyte like $25-35 \mathrm{mmol} / \mathrm{l}$ of $\mathrm{NaCl}$ solution. High salt concentration neutralizes free negative charges of citrate when chloroauric acid $\left(\mathrm{HAuCl}_{4}\right)$ is used for AuNPs synthesis that leads to the AuNPs aggregation. Upon addition of MA, MA-aptamer-modified AgNP complex is formed that can result an increase of resonance scattering at $470 \mathrm{~nm}$ with a linear relation to added MA concentration [75]. The increase of MA decreases the unreacted NGssDNA and the RRS intensity decrease linearly accompanied by color changes from red to blue. While using this method in MA detection, the results ranged between 1.89-81.89 $\mu \mathrm{g} / \mathrm{l}$ with LOD $0.98 \mu \mathrm{g} / \mathrm{l}$ determined by the NGssDNA probe [58]. For label free MA aptasensor, the AuNPs are incubated with anti-MA aptamer for $15 \mathrm{~min}$ followed by purification using centrifugation at 10,000 rpm for 5 min to obtain the aptamer-protected AuNPs. The salt concentration also influences results (Figure 3 ). The salt tolerance of aptamer-protected AuNPs should be tested using $\mathrm{NaCl}$ solutions at different concentrations. Initially, the higher concentration of salt solution results in the higher sensitivity. Conversely, it decreases the stability of intra-assay and the productivity of sensing system. Thus, the optimal concentration of $15 \mathrm{mM} \mathrm{NaCl}$ was adopted to induce the AuNPs aggregation and assure sensitivity. AuNPs should be treated because untreated ones show no color change after binding the target. However, this pretreatment doesn't intervene in detection optimization [76]. Intriguingly, while adding aptamer to AuNPs, there is no change in surface SPR absorption peak and stays $520 \mathrm{~nm}$ at UV-VIS spectra. By contrast, upon adding MA into AuNPs-aptamer solution the SPR absorbance $520 \mathrm{~nm}$ decreases up to $620 \mathrm{~nm}$ followed by color change from red wine to blue visible by naked eye or UV-VIS spectroscopy. Furthermore, it is important to take into account that the reason of color change is still surmised that the addition of a target results to the aggregation of AuNPs due to competitive binding of MA to aptamer (Figure 3). Compared to bare nanoparticles used in MA detection illustrated above such as AuNPS [63] and AgNPs [70], this method promises the reliability, accuracy and detection time of MA in milk samples because aptamer has ability to stabilize NPs in salts. In addition, the LOD of this method possesses same range of that LOD reported for biosphenol detection in water samples [77]. However, its LOD is lower probably due to the complexity of milk composition which may affect the sensitivity of target induced-AuNPs aggregation (Figure 3).

Based on colorimetric detection, the aptamer modified AuNPs probe was reported as an easy, quick, stable and selective method for MA in milk with LOD $4.2 \mu \mathrm{g} / \mathrm{l}$. Aptamers can adsorb on AuNPs surface via electrostatic forces and react with poly-diallyldimethylammonium chloride (PDDA) that prevents AuNPs aggregation. Principles of detection consist of forming MA-aptamer complex via hydrogen bonds upon addition of MA in solution and this results the change of color due to the formation of cationic polymer which can aggregate AuNPs. The produced color can be seen with naked eye without quantitative results or measured by UV-Vis spectrum [74]. However, the degree of errors can occur due to foreign substances interference or poor manipulation.

\section{Merits of Aptamer-Based Techniques}

Nanoparticles and aptamers have remarkable features of binding with analytes due to their strong localized surface plasmon resonance and high selectivity, respectively. In MA detection, these techniques and 


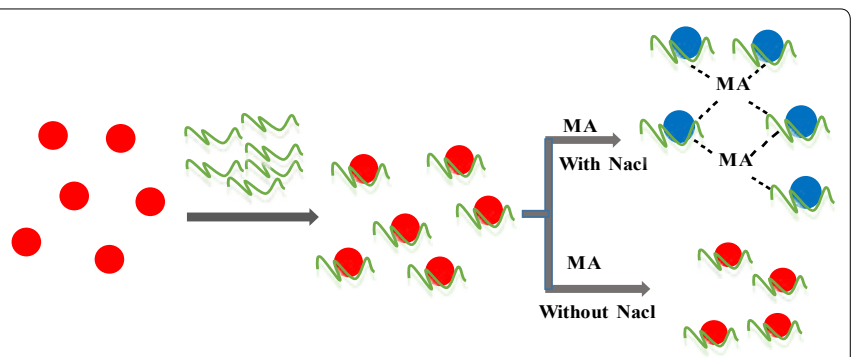

AuNPs M Aptamer

AuNPs with the color changed

Figure 3: A schematic depicting melamine detection principle using salt mediated aptamer-NPs dual system.

their combination are efficient. Comparing current research on UPLC and HPLC, MA analysis reached a concentration of $2.59 \mu \mathrm{g} / \mathrm{kg}$ using the molecular imprinted solid-phase extraction-UPLC and applied as a specific sorbent for the selective solid phase extraction of MA and its metabolites [10]. However, sample preparation, instrumentation and validation of the experiment require FTIR, NMR and/or other sophisticated techniques.

Compared to newly developed ELISA and recombinant ELISA, their detection limit ranges from $0.5-7.0 \mu \mathrm{g} / \mathrm{ml}$ [78] which is higher than those of highest aptamer-based techniques (Table 1). However, this technique can be interfered by sample matrix and also related chemical compounds, thus possibility of false results may exist. Moreover, production of monoclonal antibody for target(s) generally has several inherent limitations in vivo such as: lack of MA immunogenicity due to non- recognition by the host (only proteins and few carbohydrates have the ability to induce immune response). However, Lin et al . have developed a disclose method of producing immunogens by polymerization of non-immunogens. These methods are specific for haptens with amine and carboxylic groups including MA. Crosslinking reagents have been used to polymerize those functional groups into macromolecules to obtain haptenic polymers which will be used to immunize animal for antibodies production. The non-immunogenic MA has been polymerized using glutaraldehyde to reach a preferred complete antigen size at least of $4 \mathrm{kDa}[78,79]$. However, in vivo produced antibodies still have experimental limitations in their applications, mainly non-specific binding that can lead to cross-reactivity and high background. Therefore, aptamersrecombinant antibody can be made using library methods instead of animal immunization technologies. Additional advantages of aptamers over antibodies include easy storage, transport, suitability in variable conditions, and stability in changeable kinetic parameters, reporter molecules and high discrimination of targets. Therefore, aptamer-NPs system is a fast and more versatile approach (Table 1).

\section{Application of Aptamer in Food Safety}

As a new platform technology, aptamer-based techniques bioassay has recently been adopted in food analysis, clinical, medical and environmental monitoring [80-85]. In food safety monitoring, aptamers are applied for detection of chemical and/or biological hazards. Illegal additives used in food adulteration such as $17-\beta$ estradiol and bisphenol A were detected using DNA aptamers5'-GGGCCGTTCGAACACGAGCATGCCGGTGGGTGGTCAGGTGGGATA GCGT3' and 5'-TCCGCGAATTACACGCAGAGGTAGCGGCTCTGCGCATTCAATTGCTG-3' combined with 5'-CGCGCTGAAGCGCGGAAGC-3', respectively $[77,86,87]$. This method is based on competi- tive recognition of bisphenol with immobilized aptamer fixedon the surface of the electrode. MA detection in milk using ssDNA aptamer with sequence of 5'-TTTTTTTTTTTTTTTTTTTTTTTTTTTTTTT-3' [58] is highly effective. Aptamer detection and identification for various harmful microorganisms in clinical practice such as Pseudomonas sp. [88], Campylobacter sp. [89], Escherichia coli O157: H7 [90], Staphylococcus sp. [91], Listeria sp., and Salmonella sp. are also available [92,93]. Aptamers 5'-TTTGGTCCTTGTCTT ATGTCCAGAATGCTATGGCGGCGTCACCCGACGGG-3' and 5'-GACTTGAATTATACA GATTTCTCCTACTGGGATAGGTGGATTAT-3' sequences were used in salmonella serovars detection and showed high detection affinity. The aptamer S8-7 has been used as a ligand for magnetic capture of serially diluted Salmonella typhimurium species by detecting the whole cell content in a $290 \mu \mathrm{l}$ sample volume containing $10^{2}-10^{3} \mathrm{CFU}$ equivalents of Salmonella typhimurium which resulted in relatively high binding affinity to the target with an apparent dissociation of 1.73 $\pm 0.54 \mu \mathrm{M}$ [94]. Traditionally, Salmonella genera detection in food requires standard culture methods which include sequential steps of pre-enrichment, selective enrichment and selective differential plating. All these steps are tedious and time consuming. For decades, toxins became problematic to food safety and aptamer based techniques have been developed for the detection of toxin like Ochratoxin A with DNA aptamer [95]. Currently, Ochratoxin A has also been detected using electrochemical aptasensor [96], aflatoxins [97-100], neurotoxins [101] such as botulinum [102,103], antibiotics [104-107] as well as pesticides such as nitenpyram, chlorpyrifos and imidacloprid [108].

\section{Conclusion and Future Directives}

Aptamer, nanoparticle or aptamer-modified NPs showed efficiency in detection of MA in food or beverages with a sensitivity of 1.89$81.89 \mu \mathrm{g} / \mathrm{l}$ and $0.98 \mu \mathrm{g} / \mathrm{l}$ in short time [58]. However, AuNPs by SERS showed the concentration range of $0.31-5.0 \mathrm{mg} / \mathrm{l}$ in milk with a limit of detection of $0.17 \mathrm{mg} / \mathrm{l}$ within $30 \mathrm{~min}$ by improving time of detection but its LOD is of anxiety. These achievements are due to three major aspects: a) development of new aptamer-based analytical methods, b) modification in nanoparticles treatment and c) combination of techniques. To date many workers use aptamers for protecting and stabilizing nanoparticles. So far, the improved SELEX protocol is the promising tool for the detection of various target analytes, but it is still under continuous evolution. Theoretically, SELEX protocol is used in target analytes screening, but the market is still dominated by antibodybased method. This is due to two major reasons: 1) development of sensitive and specific aptamer for specific categories of target analytes is still limited and 2) limitations in further SELEX technology in order to produce efficient and low cost aptamer. In addition, it should be kept in mind that many of the above mentioned aptamer-based techniques are conceptual in that much works need to be done before practical application, even though it showed more stability in salts compared to techniques that use bare nanoparticles. Although aptamer-conjugated nanomaterials are emerging as promising platform for MA detection, much works remain in development of other aptamer-based methods because natural and modified natural nucleotides used during aptamers production often lack specificity and desired binding affinity. Thus, we propose aptamers development using expanded genetic code whereby natural and unnatural bases can be mixed and optimized for MA detection. Moreover, other tasks are to be done in investigating aptamer-NPs array-based, discovering combinatorial techniques beyond two as mentioned in detection of Salmonella typhimurium using nano-ELAAS (gold nanoparticles, ELISA and aptamer) [23] and developing relevant kits could curtail the time, errors and elucidate 
Citation: Omar M, Dieu NJD, Shamoon M, Lee BH (2015) Current Advances and Prospects on Implementation of Highly Sensitive Aptamer-based Dual System for Melamine Detection: New Promising Tool of Great Affinity. J Bioprocess Biotech 5: 220 doi:10.4172/2155-9821.1000220

Page 6 of 8

\begin{tabular}{|c|c|c|c|c|}
\hline Technique & Type of Food & Sensitivity & LOD & Reference \\
\hline \multirow{2}{*}{ HPLC } & Milk & $0.05-10.0 \mu \mathrm{g} / \mathrm{ml}$ & $8.1 \mu \mathrm{g} / \mathrm{l}$ & [109] \\
\hline & Edible plants & - & $2.51 \mu \mathrm{g} / \mathrm{kg}$ & [17] \\
\hline \multirow[t]{2}{*}{ GC-MS } & Milk & - & $0.01 \mathrm{mg} / \mathrm{kg}$ & [110] \\
\hline & Eggs & & $10 \mu \mathrm{g} / \mathrm{kg}$ & [102] \\
\hline LC-MS & Eggs & - & $8 \mu \mathrm{g} / \mathrm{kg}$ & {$[15,111]$} \\
\hline UPLC-MS/MS & Eggs & - & $5-10 \mu \mathrm{g} / \mathrm{kg}$ & [102] \\
\hline ELISA & $\begin{array}{l}\text { Milk, Milk products and animal } \\
\text { feeds }\end{array}$ & & $5.210-5.460 \mu \mathrm{g} / /$ & {$[79,112]$} \\
\hline ELISA KITS & $\begin{array}{l}\text { Milk, milk products and animal } \\
\text { feeds }\end{array}$ & - & $1.908,2.125,4.15 \mu \mathrm{g} / \mathrm{I}$ & [112] \\
\hline Bare AuNPs & Milk & $0.31-5.0 \mathrm{mg} / \mathrm{l}$ & $0.17 \mathrm{mg} / \mathrm{l}$ & {$[66,113]$} \\
\hline Bare AuNPs Kit & Milk products & $1-120 \mathrm{mg} / \mathrm{l}$ & - & [64] \\
\hline Bare AgNPs & Milk & $2.32-0.29 \mu \mathrm{g} / \mathrm{l}$ & - & {$[70,73]$} \\
\hline Bare Aptamer & Milk & 0.2 to $24 \mu \mathrm{g} / \mathrm{l}$ & $10.08 \mu \mathrm{g} / \mathrm{l}$ & [114] \\
\hline AuNPs-Aptamer & Milk & $1.89-81.89 \mu \mathrm{g} / \mathrm{l}$ & $0.98 \mu \mathrm{g} / \mathrm{l}$ & {$[58,59]$} \\
\hline AgNPs-Aptamer & Milk & $6.3403 .6 \mu \mathrm{g} / \mathrm{l}$ & $1.2 \mu \mathrm{g} / \mathrm{l}$ & [115] \\
\hline SAA-AgNPs & Milk & $0.1-3.1 \mu \mathrm{M}$ & $10.6 \mathrm{nM}$ & [73] \\
\hline CTA-AgNPs & Milk & $126 \mu \mathrm{g} / \mathrm{l}$ & $4.5 \mu \mathrm{g} / \mathrm{l}$ & [72] \\
\hline
\end{tabular}

Table 1: Recent reports on MA detection in food samples.

some obstacles like interference of other compounds towards high throughput detection of MA and other targets in food stuff.

\section{References}

1. Osborne CA, Lulich JP, Ulrich LK, Koehler LA, Albasan H, et al. (2009) Melamine and cyanuric acid-induced crystalluria, uroliths, and nephrotoxicity in dogs and cats. Vet Clin North Am Small Anim Pract 39: 1-14.

2. Thompson M, Owen L, Wilkinson K, Wood R, Damant A (2004) Testing for bias between the Kjeldahl and Dumas methods for the determination of nitrogen in meat mixtures, by using data from a designed interlaboratory experiment. Meat Sci 68: 631-634.

3. Yasui T, Kobayashi T, Okada A, Hamamoto S, Hirose M, et al. (2014) Longterm follow-up of nephrotoxicity in rats administered both melamine and cyanuric acid. BMC Res Notes 7: 87.

4. Jooste PJ, Anelich L, Motarjemi Y (2014) Safety of Food and Beverages: Milk and Dairy Products. In: Motarjemi Y, Moy G, Todd E (eds.) Encyclopedia of Food Safety. Academic Press, Waltham, USA, pp. 285-296.

5. Chen Y, Yang W, Wang Z, Peng Y, Li B, et al. (2010) Deposition of melamine in eggs from laying hens exposed to melamine contaminated feed. J Agric Food Chem 58: 3512-3516.

6. Moore JC, DeVries JW, Lipp M, Griffiths JC, Abernethy DR (2010) Total protein methods and their potential utility to reduce the risk of food protein adulteration. Compreh Revi Food Sci Food Safety 9: 330-357.

7. Tsai IL, Sun SW, Liao HW, Lin SC, Kuo CH (2009) Rapid analysis of melamine in infant formula by sweeping-micellar electrokinetic chromatography. J Chromatogr A 1216: 8296-8303.

8. Dai H, Shi Y, Wang Y, Sun Y, Hu J, et al. (2014) Label-free turn-on fluorescent detection of melamine based on the anti-quenching ability of $\mathrm{Hg}^{2+}$ to gold nanoclusters. Biosens Bioelectron 53: 76-81.

9. Tang G, Du L, Su X (2013) Detection of melamine based on the fluorescence resonance energy transfer between CdTe QDs and Rhodamine B. Food Chem 141: 4060-4065.

10. Ge X, Wu X, Wang J, Liang S, Sun $\mathrm{H}$ (2015) Highly sensitive determination of cyromazine, melamine, and their metabolites in milk by molecularly imprinted solid-phase extraction combined with ultra-performance liquid chromatography. J Dairy Sci 98: 2161-2171.

11. Xue J, Lee PT, Compton RG (2014) Electrochemical detection of melamine. Electroanalysis 26: 1454-1460.

12. Liu S-S, Yi X-Y, Wang J-X (2014) Sensitive detection of Melamine at surface plasmon resonance chips pre-immobilized with bovine serum albuminmelamine conjugate. Chinese J Anal Chem 42: 695-700.

13. Wu B, Wang Z, Zhao D, Lu X (2012) A novel molecularly imprinted impedimetric sensor for melamine determination. Talanta 101: 374-381.
14. Tan J, Li R, Jiang Z-T (2011) Determination of melamine in liquid milk and milk powder by titania-based ligand-exchange hydrophilic interaction liquid chromatography. Food Anal Methods 5: 1062-1069.

15. Rodriguez Mondal AM, Desmarchelier A, Konings E, Acheson-Shalom R Delatour T (2010) Liquid chromatography-tandem mass spectrometry (LC-MS/ MS) method extension to quantify simultaneously melamine and cyanuric acid in egg powder and soy protein in addition to milk products. J Agric Food Chem 58: 11574-11579.

16. Beltran-Martinavarro B, Peris-Vicente J, Carda-Broch S, Esteve-Romero J (2014) Development and validation of a micellar liquid chromatography-based method to quantify melamine in swine kidney. Food Control 46: 168-173.

17. Ge X, Wu X, Liang S, Sun H (2014) A sensitive and validated HPLC method for the determination of cyromazine and melamine in herbal and edible plants using accelerated solvent extraction and cleanup with SPE. J Chromatogr Sci 52: 751-757.

18. Zhang Y, Lin S, Jiang P, Zhu X, Ling J, et al. (2014) Determination of melamine and cyromazine in milk by high performance liquid chromatography coupled with online solid-phase extraction using a novel cation-exchange restricted access material synthesized by surface initiated atom transfer radical polymerization. J Chromatogr A 1337: 17-21.

19. Liu F, Zou MQ, Zhang M, Zhang XF, Li M (2014) Study of fast pretreatment method in detection of melamine in liquid milk using liquid chromatography and Raman spectroscopy. Guang Pu Xue Yu Guang Pu Fen Xi 34: 685-688.

20. Kong Y, Wei C, Hou Z, Wang Z, Yuan J, et al. (2014) Stacking and analysis of melamine in milk products with acetonitrile-salt stacking technique in capillary electrophoresis. J Anal Methods Chem 2014: 212697.

21. Sun F, Ma W, Xu L, Zhu Y, Liu L, et al. (2010) Analytical methods and recent developments in the detection of melamine. TrAC Trends Anal Chem 29: 1239-1249.

22. Zhou Y, Li CY, Li YS, Ren HL, Lu SY, et al. (2012) Monoclonal antibody based inhibition ELISA as a new tool for the analysis of melamine in milk and pet food samples. Food Chem 135: 2681-2686.

23. Wu W, Li J, Pan D, Li J, Song S, et al. (2014) Gold nanoparticle-based enzyme-linked antibody-aptamer sandwich assay for detection of Salmonella typhimurium. ACS Appl Mater Interfaces 6: 16974-16981.

24. Lv Z, Liu J, Bai W, Yang S, Chen A (2015) A simple and sensitive label-free fluorescent approach for protein detection based on a Perylene probe and aptamer. Biosens Bioelectron 64: 530-534.

25. Xin JY, Zhang LX, Chen DD, Lin K, Fan HC, et al. (2015) Colorimetric detection of melamine based on methanobactin-mediated synthesis of gold nanoparticles. Food Chem 174: 473-479.

26. Wu Q, Long Q, Li H, Zhang Y, Yao S (2015) An upconversion fluorescence resonance energy transfer nanosensor for one step detection of melamine in raw milk. Talanta 136: 47-53. 
Citation: Omar M, Dieu NJD, Shamoon M, Lee BH (2015) Current Advances and Prospects on Implementation of Highly Sensitive Aptamer-based Dual System for Melamine Detection: New Promising Tool of Great Affinity. J Bioprocess Biotech 5: 220 doi:10.4172/2155-9821.1000220

27. Chen L, Chen ZN (2015) A multifunctional label-free electrochemical impedance biosensor for $\mathrm{Hg}(2+)$, adenosine triphosphate and thrombin. Talanta 132: 664668.

28. Roushani M, Shahdost-fard F (2015) A novel ultrasensitive aptasensor based on silver nanoparticles measured via enhanced voltammetric response of electrochemical reduction of riboflavin as redox probe for cocaine detection. Sensors Actuators B-Chemical 207: 764-771.

29. Smith JE, Griffin DK, Leny JK, Hagen JA, Chávez JL, et al. (2014) Colorimetric detection with aptamer-gold nanoparticle conjugates coupled to an androidbased color analysis application for use in the field. Talanta 121: 247-255.

30. Ellington AD, Szostak JW (1990) In vitro selection of RNA molecules that bind specific ligands. Nature 346: 818-822.

31. Bouvet $P(2009)$ Identification of nucleic acid high-affinity binding sequences of proteins by SELEX. Methods Mol Biol 543: 139-150.

32. Yurdakok B, Filazi A, Ekici H, Celik TH, Sireli UT (2014) Melamine in breas milk. Toxicology Res 3: 242-246.

33. Li X, Lu J, Shang P, Bao J, Yue Z (2015) The selective NADPH oxidase inhibitor apocynin has potential prophylactic effects on melamine-related nephrolithiasis in vitro and in vivo. Mol Cell Biochem 399: 167-178.

34. Yang Y, Xiong GJ, Yu DF, Cao J, Wang LP, et al. (2012) Acute low-dose melamine affects hippocampal synaptic plasticity and behavior in rats. Toxicol Lett 214: 63-68.

35. Yin RH, Liu J, Li HS, Bai WL, Yin RL, et al. (2014) The toxic effects of melamine on spleen lymphocytes with or without cyanuric acid in mice. Res Vet Sci 97 505-513.

36. Yang Y, Yang D, Schluesener HJ, Zhang Z (2007) Advances in SELEX and application of aptamers in the central nervous system. Biomol Eng 24: 583-592.

37. Shimada T, Fujita N, Maeda M, Ishihama A (2005) Systematic search for the Cra-binding promoters using genomic SELEX system. Genes Cells 10: 907918

38. Jensen AE, Hjeltnes N, Berstad J, Stanghelle JK (1995) Residual urine following intermittent catheterisation in patients with spinal cord injuries. Paraplegia 33 693-696.

39. Kopylov AM, Spiridonova VA (2000) Combinatorial chemistry of nucleic acids: SELEX. Mol Biol 34: 940-954.

40. Spiridonova LN (2014) Introgression of nuclear and mitochondrial DNA markers of Mus musculus musculus to aboriginal populations of wild mice from central Asia (M. m. wagneri) and south Siberia (M. m. gansuensis). Mol Biol (Mosk) 48: $89-98$

41. Spiridonova VA, Levashov PA, Ovchinnikova ED, Afanasieva OI, Glinkina KA et al. (2014) DNA Aptamer-Based Sorbents for Binding Human IgE. Russian J Bioorganic Chem 40: 151-154.

42. Wu L, Curran JF (1999) An allosteric synthetic DNA. Nucleic Acids Res 27 1512-1516.

43. Torres-Chavolla E, Alocilja EC (2009) Aptasensors for detection of microbia and viral pathogens. Biosens Bioelectron 24: 3175-3182.

44. Kulbachinskiy AV (2007) Methods for selection of aptamers to protein targets. Biochemistry (Mosc) 72: 1505-1518.

45. Ohuchi SP, Ohtsu T, Nakamura Y (2006) Selection of RNA aptamers against recombinant transforming growth factor-beta type III receptor displayed on cell surface. Biochimie 88: 897-904

46. Chen CK (2007) Complex SELEX against target mixture: stochastic computer model, simulation, and analysis. Comput Methods Programs Biomed 87: 189200.

47. Hamula C, Guthrie J, Zhang H, Li X, Le X (2006) Selection and analytical applications of aptamers. TrAC Trends Anal Chem 25: 681-691.

48. Martell RE, Nevins JR, Sullenger BA (2002) Optimizing aptamer activity for gene therapy applications using expression cassette SELEX. Mol Ther 6: 30-34.

49. Klussmann S, Nolte A, Bald R, Erdmann VA, Fürste JP (1996) Mirror-image RNA that binds D-adenosine. Nat Biotechnol 14: 1112-1115.

50. Park HC, Baig IA, Lee SC, Moon JY, Yoon MY (2014) Development of ssDNA aptamers for the sensitive detection of Salmonella typhimurium and Salmonella enteritidis. Appl Biochem Biotechnol 174: 793-802.
51. Kasahara $Y$, Irisawa $Y$, Ozaki H, Obika S, Kuwahara M (2013) 2' 4'-BNA LNA aptamers: CE-SELEX using a DNA-based library of full-length $2^{\prime}-\mathrm{O}, 4^{\prime}-\mathrm{C}$ methylene-bridged/linked bicyclic ribonucleotides. Bioorg Med Chem Lett 23 1288-1292.

52. Lin H, Zhang W, Jia S, Guan Z, Yang CJ, et al. (2014) Microfluidic approaches to rapid and efficient aptamer selection. Biomicrofluidics 8: 041501.

53. Hünniger T, Wessels H, Fischer C, Paschke-Kratzin A, Fischer M (2014) Just in time-selection: A rapid semiautomated SELEX of DNA aptamers using magnetic separation and BEAMing. Anal Chem 86: 10940-10947.

54. Stoltenburg R, Reinemann C, Strehlitz B (2005) FluMag-SELEX as an advantageous method for DNA aptamer selection. Anal Bioanal Chem 383 : 83-91.

55. Savory N, Abe K, Yoshida W, Ikebukuro K (2014) In silico maturation: Processing sequences to improve biopolymer functions based on genetic algorithms. In Valadi J, Siarry P (eds.) Applications of Metaheuristics in Process Engineering: Springer International Publishing, USA, pp. 271-288.

56. Jarosch F, Buchner K, Klussmann S (2006) In vitro selection using a dual RNA library that allows primerless selection. Nucleic Acids Res 34: e86.

57. Kimoto M, Yamashige R, Matsunaga K, Yokoyama S, Hirao I (2013) Generation of high-affinity DNA aptamers using an expanded genetic alphabet. Nat Biotechnol 31: 453-457.

58. Liang A, Zhou L, Qin H, Zhang $Y$, Ouyang $H$, et al. (2011) A highly sensitive aptamer-nanogold catalytic resonance scattering spectral assay for melamine. J Fluoresc 21: 1907-1912.

59. Yun W, Li H, Chen S, Tu D, Xie W, et al. (2014) Aptamer-based rapid visual biosensing of melamine in whole milk. Eur Food Res Technol 238: 989-995.

60. McFarland AD, Haynes CL, Mirkin CA, Van Duyne RP, Godwin HA (2004) Citrate synthesis of gold nanoparticles. J Chem Educ 81: 544A.

61. Ai K, Liu Y, Lu L (2009) Hydrogen-bonding recognition-induced color change of gold nanoparticles for visual detection of melamine in raw milk and infant formula. J Am Chem Soc 131: 9496-9497.

62. Li C, Li D, Wan G, Xu J, Hou W (2011) Facile synthesis of concentrated gold nanoparticles with low size-distribution in water: temperature and $\mathrm{pH}$ controls. Nanoscale Res Lett 6: 440 .

63. Li L, Li B, Cheng D, Mao L (2010) Visual detection of melamine in raw milk using gold nanoparticles as colorimetric probe. Food Chem 122: 895-900.

64. Zhou Q, Liu N, Qie Z, Wang Y, Ning B, et al. (2011) Development of gold nanoparticle-based rapid detection kit for melamine in milk products. J Agric Food Chem 59: 12006-12011.

65. Cao Q, Zhao H, He Y, Li X, Zeng L, et al. (2010) Hydrogen-bonding-induced colorimetric detection of melamine by nonaggregation-based Au-NPs as a probe. Biosens Bioelectron 25: 2680-2685.

66. Kumar N, Seth R, Kumar H (2014) Colorimetric detection of melamine in milk by citrate-stabilized gold nanoparticles. Anal Biochem 456: 43-49.

67. Giovannozzi AM, Rolle F, Sega M, Abete MC, Marchis D, et al. (2014) Rapid and sensitive detection of melamine in milk with gold nanoparticles by Surface Enhanced Raman Scattering. Food Chem 159: 250-256.

68. Wagner J, Tshikhudo TR, Kohler JM (2008) Microfluidic generation of meta nanoparticles by borohydride reduction. Chem Eng J 135: S104-S109.

69. Pinto VV, Ferreira MJ, Silva R, Santos HA, Silva F, et al. (2010) Long time effect on the stability of silver nanoparticles in aqueous medium: Effect of the synthesis and storage conditions. Colloids and Surfaces A: Physicochem Eng Aspects 364: 19-25.

70. Ping H, Zhang M, Li H, Li S, Chen Q, et al. (2012) Visual detection of melamine in raw milk by label-free silver nanoparticles. Food Control 23: 191-197.

71. Buka I, Osornio-Vargas A, Karr C (2009) Melamine food contamination: Relevance to Canadian children. Paediatr Child Health 14: 222-224.

72. Song J, Wu F, Wan Y, Ma LH (2014) Visual test for melamine using silve nanoparticles modified with chromotropic acid. Microchimica Acta 181: 12671274.

73. Song J, Wu F, Wan Y, Ma L (2014) Colorimetric detection of melamine in pretreated milk using silver nanoparticles functionalized with sulfanilic acid. Food Control 50: 356-361. 
Citation: Omar M, Dieu NJD, Shamoon M, Lee BH (2015) Current Advances and Prospects on Implementation of Highly Sensitive Aptamer-based Dual System for Melamine Detection: New Promising Tool of Great Affinity. J Bioprocess Biotech 5: 220 doi:10.4172/2155-9821.1000220

74. Xing HB, Zhan SS, Wu YG, He L, Zhou P (2013) Sensitive colorimetric detection of melamine in milk with an aptamer-modified nanogold probe. RSC Adv 3: 17424-17430

75. Liang A, Zhou L, Jiang Z (2011) A simple and sensitive resonance scattering spectral assay for detection of melamine using aptamer-modified nanosilver probe. Plasmonics 6: 387-392.

76. Mei Z, Chu H, Chen W, Xue F, Liu J, et al. (2013) Ultrasensitive one-step rapid visual detection of bisphenol $\mathrm{A}$ in water samples by label-free aptasensor. Biosens Bioelectron 39: 26-30.

77. Zhou L, Wang J, Li D, Li Y (2014) An electrochemical aptasensor based on gold nanoparticles dotted graphene modified glassy carbon electrode for label-free detection of bisphenol A in milk samples. Food Chem 162: 34-40.

78. Li W, Meng M, Lu X, Liu W, Yin W, et al. (2014) Preparation of anti-melamine antibody and development of an indirect chemiluminescent competitive ELISA for melamine detection in milk. Food Agric Immunol 25: 498-509.

79. Cao B, Yang H, Song J, Chang H, Li S, et al. (2013) Sensitivity and specificity enhanced enzyme-linked immunosorbent assay by rational hapten modification and heterogeneous antibody/coating antigen combinations for the detection of melamine in milk, milk powder and feed samples. Talanta 116: 173-180.

80. Tombelli S, Minunni M, Mascini M (2007) Aptamers-based assays for diagnostics, environmental and food analysis. Biomol Eng 24: 191-200.

81. Xu S, Yuan H, Chen S, Xu A, Wang J, et al. (2012) Selection of DNA aptamers against polychlorinated biphenyls as potential biorecognition elements for environmental analysis. Anal Biochem 423: 195-201.

82. Centi S, Silva E, Laschi S, Palchetti I, Mascini M (2007) Polychlorinated biphenyls (PCBs) detection in milk samples by an electrochemical magnetoimmunosensor (EMI) coupled to solid-phase extraction (SPE) and disposable low-density arrays. Anal Chim Acta 594: 9-16.

83. Citartan M, Gopinath SC, Tominaga J, Tan SC, Tang TH (2012) Assays for aptamer-based platforms. Biosens Bioelectron 34: 1-11.

84. Kim SE, Su W, Cho M, Lee Y, Choe WS (2012) Harnessing aptamers for electrochemical detection of endotoxin. Anal Biochem 424: 12-20.

85. Xu S, Yuan H, Chen S, Xu A, Wang J, et al. (2012) Selection of DNA aptamers against polychlorinated biphenyls as potential biorecognition elements for environmental analysis. Anal Biochem 423: 195-201.

86. Marks HL, Pishko MV, Jackson GW, Coté GL (2014) Rational design of a bisphenol A aptamer selective surface-enhanced Raman scattering nanoprobe. Anal Chem 86: 11614-11619.

87. Yildirim N, Long F, He M, Shi HC, Gu AZ (2014) A portable optic fibe aptasensor for sensitive, specific and rapid detection of bisphenol-A in water samples. Environ Sci Process Impacts 16: 1379-1386.

88. Kim LH, Yu HW, Kim YH, Kim IS, Jang A (2013) Potential of fluorophore labeled aptamers for Pseudomonas aeruginosa detection in drinking water. J Korean Soc Appl Biol Chem 56: 165-171.

89. Suh SH, Dwivedi HP, Jaykus LA (2014) Development and evaluation of aptamer magnetic capture assay in conjunction with real-time PCR for detection of Campylobacter jejuni. Lwt-Food Sci Technol 56: 256-260.

90. Wu W, Zhao S, Mao Y, Fang Z, Lu X, et al. (2015) A sensitive lateral flow biosensor for Escherichia coli O157:H7 detection based on aptamer mediated strand displacement amplification. Anal Chim Acta 861: 62-68.

91. Chang YC, Yang CY, Sun RL, Cheng YF, Kao WC, et al. (2013) Rapid single cell detection of Staphylococcus aureus by aptamer-conjugated gold nanoparticles. Sci Rep 3: 1863.

92. Liu G, Lian Y, Gao C, Yu X, Zhu M, et al. (2014) In vitro selection of DNA aptamers and fluorescence-based recognition for rapid detection Listeria monocytogenes. J Integrative Agric 13: 1121-1129.

93. Suh SH, Dwivedi HP, Choi SJ, Jaykus LA (2014) Selection and characterization of DNA aptamers specific for Listeria species. Anal Biochem 459: 39-45.

94. Dwivedi HP, Smiley RD, Jaykus LA (2013) Selection of DNA aptamers for capture and detection of Salmonella typhimurium using a whole-cell SELEX approach in conjunction with cell sorting. Appl Microbiol Biotechnol 97: 36773686.

95. Cruz-Aguado JA, Penner G (2008) Determination of ochratoxin a with a DNA aptamer. J Agric Food Chem 56: 10456-10461.
96. Wu J, Chu H, Mei Z, Deng Y, Xue F, et al. (2012) Ultrasensitive one-step rapid detection of ochratoxin $A$ by the folding-based electrochemical aptasensor. Anal Chim Acta 753: 27-31.

97. Ali WH, Pichon V (2014) Characterization of oligosorbents and application to the purification of ochratoxin A from wheat extracts. Anal Bioanal Chem 406: 1233-1240.

98. Luan Y, Chen Z, Xie G, Chen J, Lu A, et al. (2015) Rapid visual detection of aflatoxin $B 1$ by label-free aptasensor using unmodified gold nanoparticles. $J$ Nanosci Nanotechnol 15: 1357-1361.

99. Ma X, Wang W, Chen X, Xia Y, Duan N, et al. (2015) Selection, characterization and application of aptamers targeted to Aflatoxin B2. Food Control 47: 545-551.

100.Shim WB, Mun H, Joung HA, Ofori JA, Chung DH, et al. (2014) Chemiluminescence competitive aptamer assay for the detection of aflatoxin B1 in corn samples. Food Control 36: 30-35.

101. McConnell EM, Holahan MR, DeRosa MC (2014) Aptamers as promising molecular recognition elements for diagnostics and therapeutics in the central nervous system. Nucleic Acid Ther 24: 388-404.

102. Xia X, Ding S, Li X, Gong X, Zhang S, et al. (2009) Validation of a confirmatory method for the determination of melamine in egg by gas chromatographymass spectrometry and ultra-performance liquid chromatography-tandem mass spectrometry. Anal Chim Acta 651: 196-200.

103. Chang TW, Blank M, Janardhanan P, Singh BR, Mello C, et al. (2010) In vitro selection of RNA aptamers that inhibit the activity of type A botulinum neurotoxin. Biochem Biophys Res Commun 396: 854-860.

104.Wang K, Tao ZH, Xu L, Liu YQ (2014) Research and development of functionalized aptamer based biosensor. Chinese J Anal Chem 42: 298-304.

105. Ma L, Wen GQ, Liu QY, Liang AH, Jiang ZL (2014) Resonance Rayleigh scattering determination of trace tobramycin using aptamer-modified nanogold as probe. Guang Pu Xue Yu Guang Pu Fen Xi 34: 2481-2484.

106. Lin Z, Ma Q, Fei X, Zhang H, Su X (2014) A novel aptamer functionalized CulnS2 quantum dots probe for daunorubicin sensing and near infrared imaging of prostate cancer cells. Anal Chim Acta 818: 54-60.

107. Yan L, Luo C, Cheng W, Mao W, Zhang D, et al. (2012) A simple and sensitive electrochemical aptasensor for determination of Chloramphenicol in honey based on target-induced strand release. J Electroanal Chem 687: 89-94.

108. He J, Liu Y, Fan M, Liu X (2011) Isolation and identification of the DNA aptame target to acetamiprid. J Agric Food Chem 59: 1582-1586.

109. Finete Vde L, Gouvêa MM, Marques FF, Netto AD (2014) Characterization of newfound natural luminescent properties of melamine, and development and validation of a method of high performance liquid chromatography with fluorescence detection for its determination in kitchen plastic ware. Talanta 123: $128-134$

110. Pan XD, Wu P, Yang DJ, Wang LY, Shen XH, et al. (2013) Simultaneous determination of melamine and cyanuric acid in dairy products by mixed-mode solid phase extraction and GC-MS. Food Control 30: 545-548.

111. Wang PC, Lee RJ, Chen CY, Chou CC, Lee MR (2012) Determination of cyromazine and melamine in chicken eggs using quick, easy, cheap, effective, rugged and safe (QuEChERS) extraction coupled with liquid chromatographytandem mass spectrometry. Anal Chim Acta 752: 78-86.

112. Gong Y, Zhang M, Wang M, Chen Z, Xi X (2014) Development of immunobased methods for detection of melamine. Arabian J Sci Eng 39: 5315-5324.

113. Giovannozzi AM, Rolle F, Sega M, Abete MC, Marchis D, et al. (2014) Rapid and sensitive detection of melamine in milk with gold nanoparticles by Surface Enhanced Raman Scattering. Food Chem 159: 250-256.

114. Wang G, Zhu Y, He X, Chen L, Wang L, et al. (2013) Colorimetric and visual determination of melamine by exploiting the conformational change of hemin G-quadruplex-DNAzyme. Microchim Acta 181: 411-418.

115. Wen G, Zhou L, Li T, Liang A, Jiang Z (2012) A sensitive surface-enhanced Raman scattering method for determination of melamine with aptamermodified nanosilver probe. Chinese J Chem 30: 869-874. 